\title{
Corrigendum
}

\section{Corrigendum to "Numerical Simulation of Droplet Impacting and Sliding on Hydrophobic Granular Surfaces"}

\author{
Qing Bao' ${ }^{1}$ and Hengyi Kang ${ }^{2}$ \\ ${ }^{1}$ School of Cyberspace, Hangzhou Dianzi University, Hangzhou 310018, Zhejiang, China \\ ${ }^{2}$ Transportation and Municipal Engineering Institute, PowerChina Huadong Engineering Corporation Limited, \\ Hangzhou 311122, Zhejiang, China
}

Correspondence should be addressed to Hengyi Kang; hykang91@hotmail.com

Received 16 October 2021; Accepted 16 October 2021; Published 28 October 2021

Copyright ( 92021 Qing Bao and Hengyi Kang. This is an open access article distributed under the Creative Commons Attribution License, which permits unrestricted use, distribution, and reproduction in any medium, provided the original work is properly cited.

In the article titled "Numerical Simulation of Droplet Impacting and Sliding on Hydrophobic Granular Surfaces" [1], the Acknowledgments section should read as follows:

\section{Acknowledgments}

Financial support provided by the General Research Fund, Research Grants Council, Hong Kong (Grant nos. 17205915, 17221016, 17203417, and T22-603/15-N) and the Hong Kong University (HKU) Seed Funding Programme for Basic Research (Grant no. 201406159004) is acknowledged. The Postgraduate Scholarship (PGS) provided by HKU is also acknowledged. This work was also supported by the National Natural Science Foundation of China (Grant no. 61806061).

\section{References}

[1] Q. Bao and H. Kang, "Numerical simulation of droplet impacting and sliding on hydrophobic granular surfaces," Mathematical Problems in Engineering, vol. 2021, Article ID 5534003, 13 pages, 2021. 